\title{
Segmentation of Diffuse Lung Abnormality Patterns on Computed Tomography Images using Partially Supervised Learning
}

\author{
Yuki SuzukI, ${ }^{*}$ Shoji KIdo, ${ }^{*}$, Shingo Mabu, ${ }^{* *}$ Masahiro YanagawA, ${ }^{* * *}$ Noriyuki TomiYAma, ${ }^{* * *}$ \\ Yoshinobu SATO ${ }^{\dagger}$
}

\begin{abstract}
Computer-aided diagnostic methods that provide semantic segmentation of texture patterns of diffuse lung diseases (DLDs) on chest computed tomography (CT) are extremely useful for detecting, identifying, and quantifying lung pathologies. While a fully annotated dataset is desirable to build a semantic segmentation model, building such a dataset for DLDs is costly due to the requirements of manual segmentation and certified experts for annotation. Partially supervised learning (PSL) has been proposed recently to take advantage of the partially annotated dataset and reduce the full annotation burden. Creating a partially annotated dataset is much less expensive than creating a fully annotated dataset. Therefore, PSL has great potential to build a semantic segmentation model that only requires a feasible amount of annotation. In this study, we propose a method of PSL employing a loss function that uses both annotated and unannotated pixels of a partially annotated dataset. The proposed loss function is based on the cross entropy loss, and it uses unannotated pixels to penalize the leakage of the segmentation. A parameter that controls the balance between the two types of supervision is introduced into the loss function to allow tuning and studying of the proposed PSL. The effectiveness and characteristics of PSL for the segmentation of DLD classes (consolidation, ground grass opacity, honeycombing, emphysema, and normal) were investigated in experiments using chest CT images of 372 patients. The experimental results show that the proposed PSL improved the mean Dice score from 0.76 to 0.79 , and that a higher value of the balancing parameter increased the precision of the segmentation. Using the proposed PSL, which takes full advantage of the partially annotated dataset, we improved the accuracy of DLD segmentation. Furthermore, the experimental results clarified that the proposed PSL improved the precision of the models using unannotated pixels. Our implementation of the proposed PSL is available at https://github.com/yk-szk/psldld.
\end{abstract}

Keywords: diffuse lung disease, partial annotation, partial supervision.

Adv Biomed Eng. 11: pp. 25-36, 2022.

Received on October 19, 2021; revised on December 15, 2021; accepted on December 27, 2021.

*Department of Artificial Intelligence Diagnostic Radiology, Osaka University Graduate School of Medicine, Osaka, Japan.

** Graduate School of Sciences and Technology for Innovation, Yamaguchi University, Yamaguchi, Japan.

*** Department of Diagnostic and Interventional Radiology, Osaka University Graduate School of Medicine, Osaka, Japan.

${ }^{\dagger}$ Division of Information Science, Graduate School of Science and Technology, Nara Institute of Science and Technology, Nara, Japan.

\# 2-2 Yamadaoka, Suita, Osaka 565-0871, Japan.

E-mail: kido@radiol.med.osaka-u.ac.jp

\section{(c) BY}

Copyright: (02022 The Author(s). This is an open access article distributed under the terms of the Creative Commons BY 4.0 International (Attribution) License (https:// creativecommons.org/licenses/by/4.0/legalcode), which permits the unrestricted distribution, reproduction and use of the article provided the original source and authors are credited.

\section{Introduction}

Diffuse lung abnormality patterns are texture patterns observed on medical images such as chest X-ray and computed tomography (CT) of patients with diffuse lung diseases (DLDs). Accurate identification of the texture patterns of DLDs is a challenging task even for experienced radiologists [1,2], and quantification of the volume and distribution of DLDs on a chest CT image is time-consuming because DLDs typically spread over a large area and numerous slices in the lung. Therefore, developing computer-aided diagnosis (CAD) system capable of promptly and objectively assessing lungs with DLDs is of great value. CAD tools for DLDs have been developed for many tasks including classifying CT slices [3], classifying image patches [4, 5], semantic segmentation at pixel level [6] and prognostic prediction [7].

While machine learning is required for the develop- 
ment of a CAD system, it frequently necessitates the use of annotated datasets, which are not always readily available. Fully convolutional networks [8, 9] (FCNs) are commonly used for semantic segmentation tasks, and FCN models are trained using a fully annotated dataset, in which every pixel of the images in the dataset is annotated. Creating a fully annotated dataset for semantic segmentation is expensive because manual segmentation of images is time-consuming. It is more expensive to create a dataset for DLDs since it requires qualified professionals (such as radiologists) and it is difficult to distinguish between different DLD texture patterns with normal and unusual appearances. Therefore, creating a large-scale fully annotated dataset for DLDs is almost impossible.

In most cases, there is a trade-off between accuracy and the amount of annotated dataset used for training machine learning. Unsupervised learning is at one end of the trade-off spectrum because it does not require an annotated dataset. However, its accuracy is not as good as supervised methods because no supervision is provided during the training [10]. Fully supervised learning, on the other hand, is at the opposite end of the trade-off spectrum with the best performance of all learning methods. However, it requires a fully annotated dataset. Therefore, learning methods with adequate performance and minimum amount of annotation are sought to build a practical CAD system for DLDs. Partially supervised learning (PSL) [11] has recently been proposed to take advantage of a partially annotated dataset. PSL is a promising technique for DLD segmentation, because it only requires partial annotation while supervising effective training. Therefore, we focus on this learning technique in this paper.

Other than PSL, several strategies have been developed to reduce the need for annotated dataset [12]. Unannotated data approaches such as entropy minimization [13] and consistency training are used in semisupervised learning [14]. Weakly supervised learning [15] allows semantic segmentation to be performed using weak annotation such as image-level annotation $[16,17]$. Transfer learning, also known as finetuning [19], is a strategy of pre-training a model with a large-scale dataset to achieve a well-generalized model even with a limited target dataset $[20,21]$ for the domain of application. PSL and these approaches are not mutually exclusive and can be used together where appropriate.

PSL has been effectively used in various applications including abdominal organ segmentation [22-24] and brain tissue segmentation [11,25-27]. More recently, PSL has been applied to segmentation of tumors and other disease regions, which are more complicated than normal organ regions $[28,29]$. However, no application to DLDs has been reported. Applying PSL to DLDs can be more effective than applying it to normal anatomical structures such as organs and the brain, because of the differences in characteristics between DLDs and normal anatomical structures. First, DLDs may appear anywhere in the lung, making it more difficult to split input images into regions of interest (ROIs) and apply a specific model to each ROI. Second, a typical patient with DLD only has a couple of DLD lesions, unlike organ or tissue segmentation tasks, where a patient has a complete set of organs without missing any. This makes it more difficult to construct an ensemble model composed of partial models using subsets of the dataset, because each model cannot learn DLD classes absent in the subsets.

Our contribution is two-fold: (1) proposing a method of PSL that uses both annotated and unannotated pixels and applying it to DLD to demonstrate the applicability of PSL; and (2) studying the characteristics of the proposed method by introducing a parameter that controls the balance of the supervision. Although we previously presented early results [30], in this study, we performed additional evaluation using samples of fully annotated images as well as tested a wider range of the balancing parameter.

The DLD patterns including pattern of healthy lung examined in this paper were consolidation $(\mathrm{CON})$, ground-glass opacity (GGO), honeycombing (HCM), emphysema (EMP), and normal (NOR). Throughout this paper, each DLD pattern is represented or superposed in the following colors (CON: cyan, GGO: yellow, HCM: red, EMP: green, NOR: brown). The paper is organized as follows. In the Materials and Methods section, partially annotated datasets and PSL are explained. The results of the experiments using chest CT images of 372 patients are shown in the Results section. The Discussion section discusses the advantages and characteristics of the proposed method based on the experimental findings.

\section{Materials and Methods}

\subsection{Materials}

The dataset used in this paper consisted of chest CT images with a matrix size of $512 \times 512$ taken in Yamaguchi University Hospital, Japan. CT images of 372 patients were collected. The pixel size was $0.684 \pm 0.0517 \mathrm{~mm}$ (mean \pm standard deviation), and slice thickness was $1 \mathrm{~mm}$. In the experiments, no pixel size equalization was performed because the deviation in pixel size was negligibly small.

Only one class or a subset of potential classes for an image can be annotated in a partially annotated dataset. Figure 1 shows a comparison between full annotation and partial annotation. Figure 1(a) shows a fully annotated image, in which every pixel in the lung is labeled 
with one of the possible classes: $L_{\text {positive }}=\{C O N, G G O$, $H C M, E M P, N O R\}$. Figure 1(b) and (c) show partial annotation and the annotated pixels of an image, in which just one class $(\mathrm{CON})$ in the image was chosen and annotated, while other regions with other classes (GGO or NOR) were left unannotated. In Fig. 1(b), the delineated region indicates the $\mathrm{CON}$ region, and the derived annotated pixels are shown in Fig. 1(c). Partial annotation is easier and takes less time than full annotation, because with partial annotation, annotators can focus on only one label, and the area required for annotation is much smaller. Figure 1(d) shows an unannotated region of a partially annotated image. In this image, regions that are not $\mathrm{CON}$ are left unannotated, and therefore is virtually annotated as $\neg \mathrm{CON}=\{\mathrm{GGO}, \mathrm{HCM}, \mathrm{EMP}, \mathrm{NOR}\}$ region. Note that the fully annotated image shown in Fig. 1(a) is created only for illustration, and a fully annotated image like this is not available for training in this study. Although a subset of possible classes can be annotated, we only annotated one class per image in this paper.

Our partially annotated dataset was created by the following steps. First, up to three slices from each CT scan were chosen for annotation. Second, for each slice, one representing DLD class was chosen by a boardcertified radiologist. Third, three board-certified radiologists performed partial annotation of the chosen DLD class for every image. Finally, pixels annotated by two or more radiologists were designated as annotated pixels, whereas pixels that were annotated by less than two radiologists were designated unannotated. Note that three radiologists performed annotations to avoid creating a biased dataset. In this paper, unannotated pixels are expressed as one of the following labels: $L_{\text {negative }}=\{\neg C O N$, $\neg G G O, \neg H C M, \neg E M P, \neg N O R\}$. In addition to the DLD annotation, lung fields were manually segmented under the supervision of radiologists, and training and evaluation were conducted specifically within the lung fields. Figure 2 shows examples of partially annotated images for each class of DLD. Table 1 shows the statistics of the partially annotated dataset used in the experiments. In Table 1, the number of unannotated pixels for each class is the number of pixels of the corresponding negative labels. For example, the number unannotated pixels for $\mathrm{CON}$ is the number of $\neg \mathrm{CON}$ pixels.

\subsection{Methods}

The fundamental idea of the proposed PSL is to use unannotated pixels to loosely supervise the model. For example, in Fig. 1(d), the unannotated region can supervise the model so as not to output the CON label within the unannotated region. This strategy offers an advantage for the proposed PSL over other training methods that only use annotated pixels. Although unannotated pixels are less informative than annotated pixels, using unannotated pixels approximately doubles the number of pixels involved in the training, as shown in Table 1. Note that the total number of cases (405) in Table $\mathbf{1}$ is greater than the number of patients (372), because multiple slices from one patient may be chosen for annotation of different classes of DLD. Figure 3 shows an overview of the

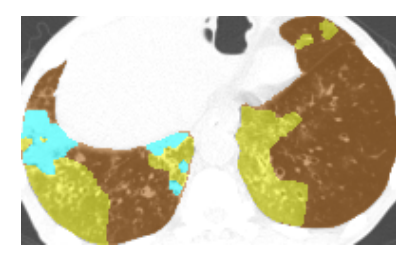

(a) Full annotation

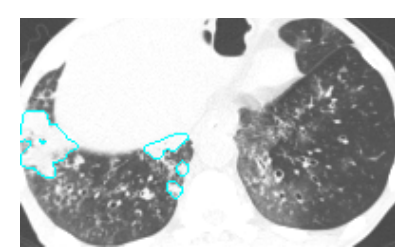

(b) Partial annotation

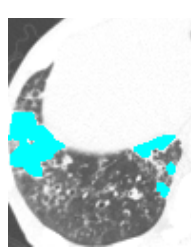

(c) Annotated (positive) pixels

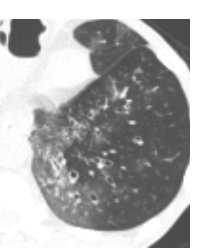

(d)

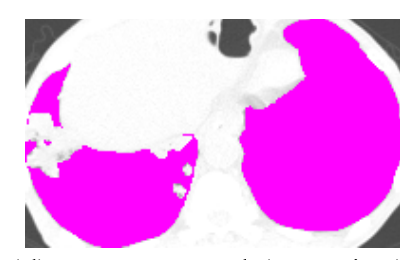

d) Unannotated (negative) pixels

Fig. 1 Comparison of fully and partially annotated images. (a) Full annotation for consolidation (CON), ground-glass opacity (GGO) and normal (NOR) regions in the lung is shown. (b) Partial annotation in which only the CON region is annotated (shown in cyan). (c) Annotated pixels (shown in cyan) derived from (b). (d) Unannotated pixels (shown in magenta), which are implicitly annotated as $\neg \mathrm{CON}$ in (b).

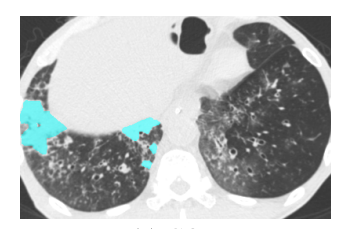

(a) $\mathrm{CON}$

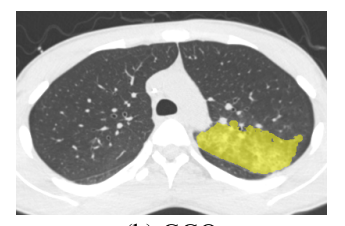

(b) $\mathrm{GGO}$

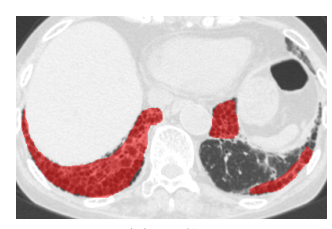

(c) $\mathrm{HCM}$

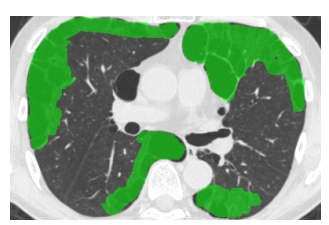

(d) EMP

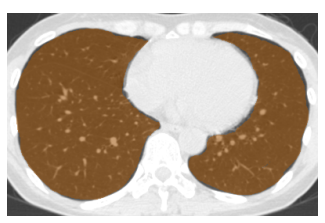

(e) NOR

Fig. 2 Typical slice of each diffuse lung disease (DLD) class. Annotated labels in transparent colors are superposed on CT slices. 
Table 1 Dataset statistics. Note that the total number of cases in the table is more than the number of cases because multiple slices with different DLD classes were extracted from one patient.

\begin{tabular}{lrrrrrr}
\hline & CON & GGO & HCM & EMP & NOR & Total \\
\hline Cases & 56 & 112 & 51 & 131 & 55 & 405 \\
Slices & 150 & 114 & 129 & 163 & 55 & 611 \\
Annotated (positive) pixels $\left(\times 10^{3}\right)$ & 658 & 1,676 & 1,345 & 4,134 & 2,552 & 10,365 \\
Unannotated (negative) pixels $\left(\times 10^{3}\right)$ & 3,878 & 2,648 & 2,405 & 2,710 & 117 & 11,759 \\
\hline
\end{tabular}

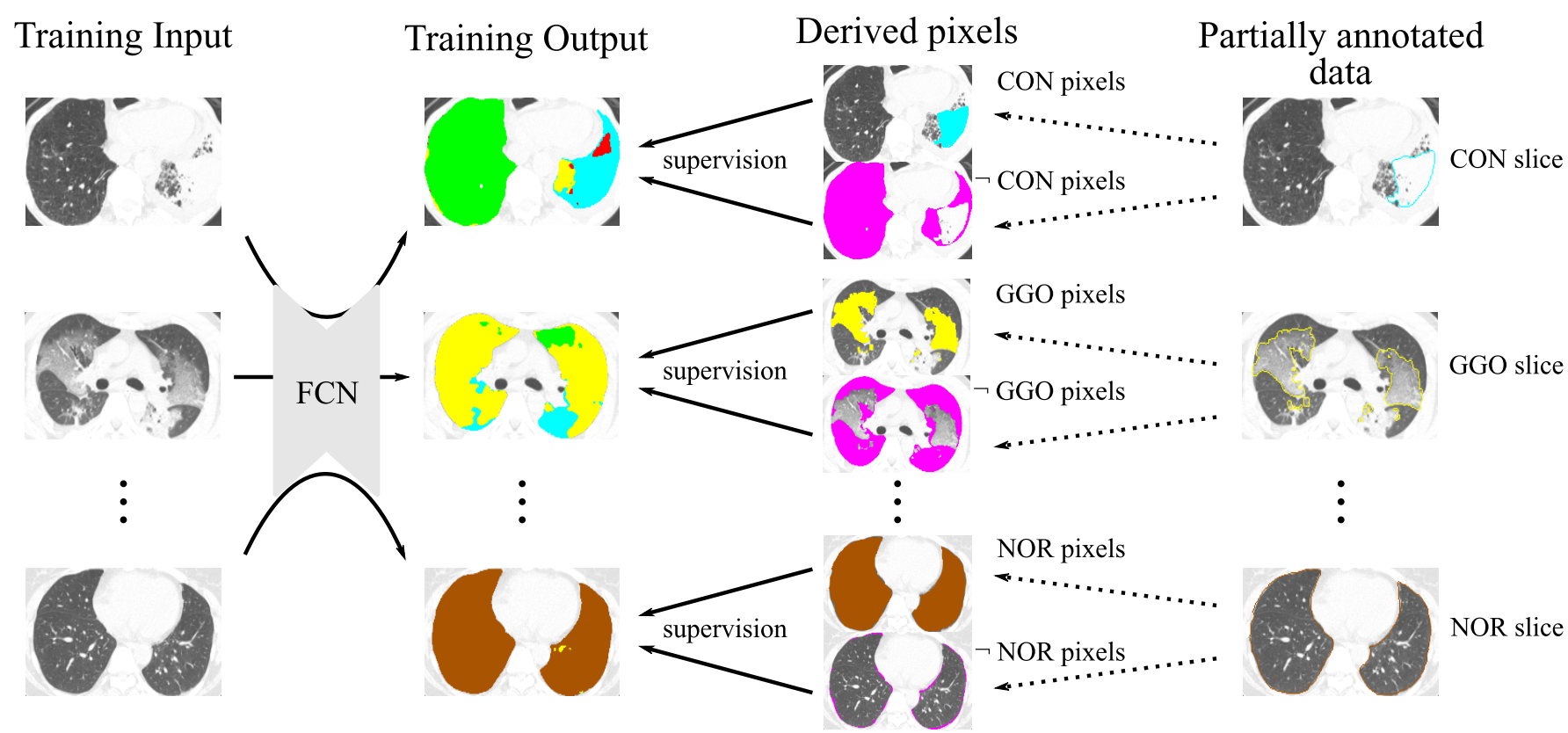

Fig. 3 Overview of the training process of the proposed partially supervised learning.

proposed PSL. As shown in Fig. 3, two types of supervision; one using annotated pixels and the other using unannotated pixels, are applied to the model.

Using unannotated pixels is realized by the loss function defined as

$$
\mathcal{L}(l, \hat{\boldsymbol{y}})= \begin{cases}(1-\lambda) * H(e(l), \hat{\boldsymbol{y}}), & l \in L_{\text {positive }} \\ \lambda * \frac{1}{H(e(l), \hat{\boldsymbol{y}})}, & l \in L_{\text {negative }}\end{cases}
$$

where $l, \hat{\boldsymbol{y}}$, and $H($.$) denote the ground-truth label, Soft-$ Max output of the model, and cross-entropy, respectively. $e(l)$ is one-hot encoding function that works regularly for $l \in L_{\text {positive }}\left(\right.$ e.g., $\left.e\left(l_{C O N}\right)=[1,0,0,0,0]\right)$, while it works for $l \in L_{\text {negative }}$ so that unannotated pixels are encoded in the same way as the corresponding annotated pixels (e.g., $\left.e\left(l_{C O N}\right)=e\left(l_{\neg C O N}\right)\right)$. This loss function is based on cross-entropy loss, and works regularly for annotated pixels, whereas it penalizes unannotated pixels for being predicted as the corresponding annotated labels. $\lambda$ is a balancing parameter that modifies the balance of the supervision used for the loss of unannotated pix- els. The proposed method with the parameter $\lambda=0$ is equivalent to the training method that only uses annotated pixels.

PSL can be used with any segmentation model and is not dependent on any network architecture. In this study, we used a slab U-Net as shown in Fig. 4 to take advantage of three-dimensional information of DLDs. The slab U-Net is a hybrid of 2D and 3D U-Nets [9, 31]. The input of the network is a $3 \mathrm{D}$ slab around an annotated slice with the shape of $6 \times 512 \times 512 \times 1$ and the output shape is $1 \times 512 \times 512 \times 5$, where elements represent the sizes along the $z, y, x$, and channel axes. In this study, $x, y$ and $z$ axes represent frontal, sagittal and longitudinal axes, respectively. Shallower layers of the network consisted of 3D operations such as 3D convolution and 3D max pooling with no padding applied to z-axis, which reduced the data size along $\mathrm{z}$-axis. Operations were switched to $2 \mathrm{D}$ after the data size along $\mathrm{Z}$-axis was reduced to one.

As data augmentation, geometric transformations (random flip around $\mathrm{y}$-axis, random rotation around 


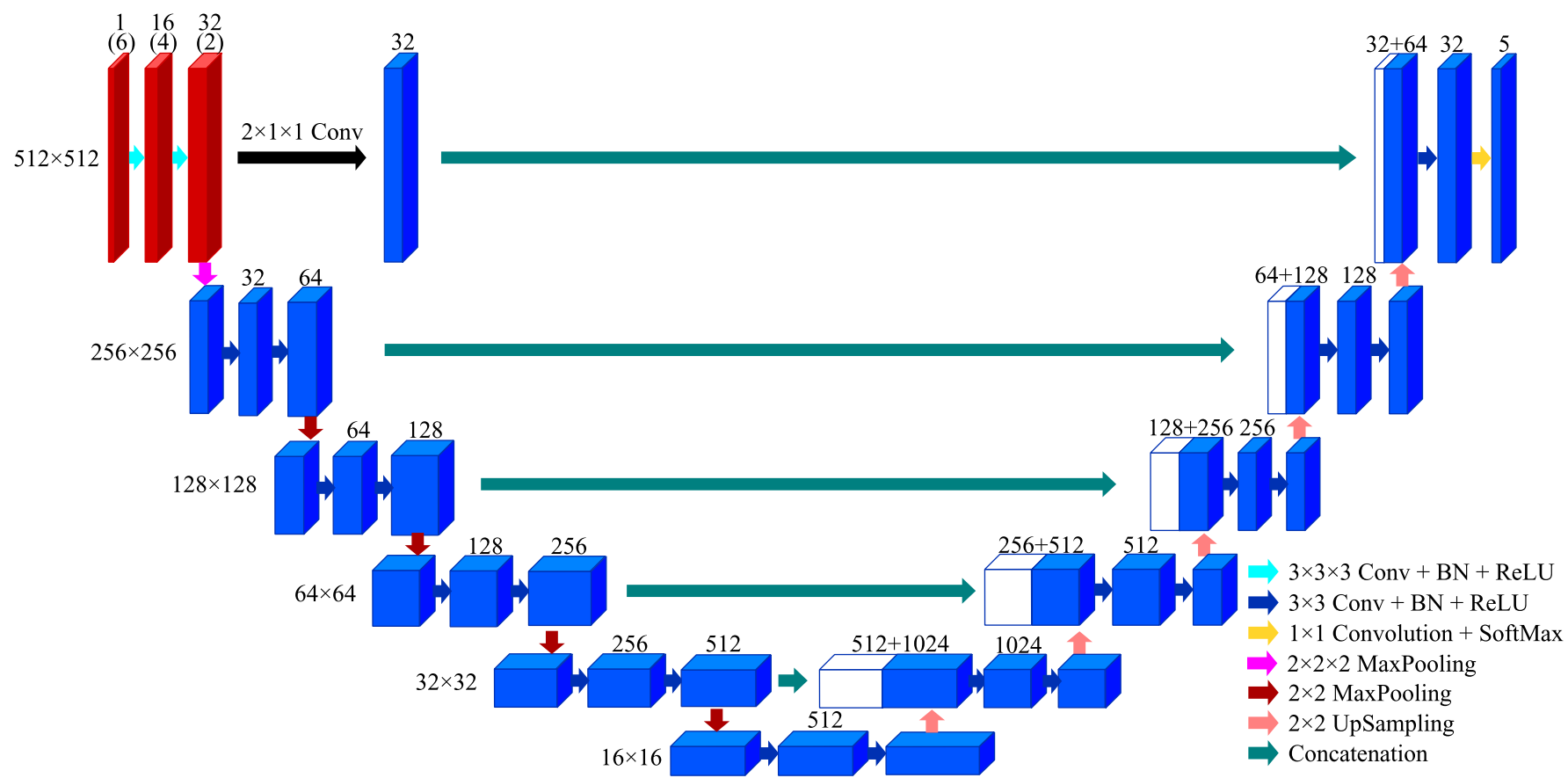

Fig. 4 The slab U-Net. The red and blue boxes represent three-dimensional and two-dimensional data, respectively. The number of channels is indicated by the numbers above the boxes. The parenthesized numbers above the red boxes indicate the sizes along z-axis. The white boxes represent concatenated data. At each level of the network, image resolution is indicated on the left side.

$\mathrm{z}$-axis from -15 to 15 degrees, and random rescaling from $90 \%$ to $110 \%$ ) are applied randomly to the input images on the fly during the training. These geometric transformations are applied primarily to increase the variation of body sizes and orientations in the training dataset but also to help the network to learn more robust feature extractors [32].

\section{RESULTS}

For training and evaluation, five-fold stratified crossvalidation was used. The stratified splitting strategy was adopted to avoid skewed results due to uneven split of the dataset, and patient-based splitting was used to avoid data leakage. Each training subset had a validation subset to determine the number of epochs using early stopping. Model network weights were optimized using Adam [33] optimizer with default parameters, and losses were computed using Eq. 1. Our model was built using TensorFlow, and the loss function and network implementation can be found at https://github.com/yk-szk/ psl-dld. As evaluation metrics, Dice similarity score, precision, and recall are calculated for each slice. The training and evaluation were performed on an NVIDIA RTX 3090 with 24 GB of graphics processing unit memory.

Figure 5 shows line plots of evaluation metrics (Dice score, precision, and recall) for the $\lambda$ values ranging from 0 to 1 with step size 0.1 . The mean Dice score and recall for $\lambda=1$ were very low $(0.079$ and 0.088 , re-

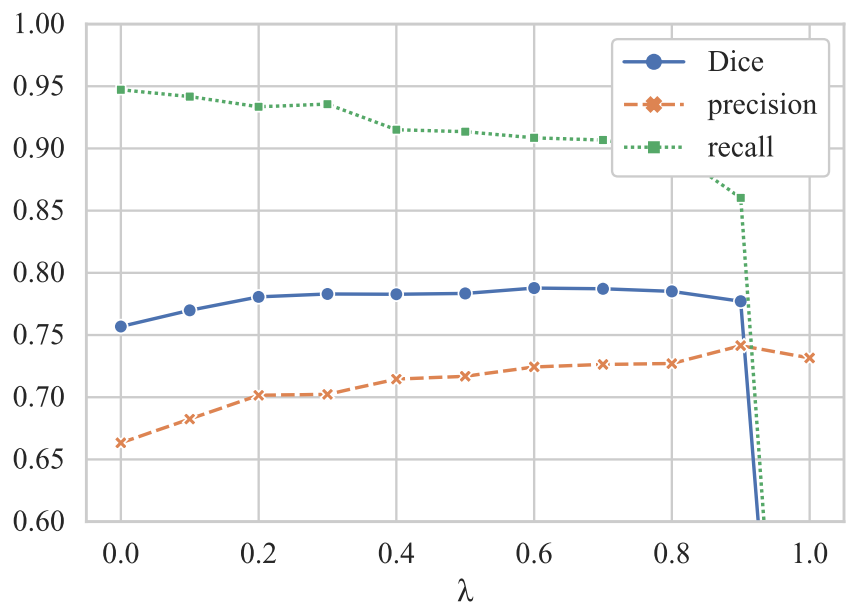

Fig. 5 Line plots of Dice score, precision and recall.

spectively) and plot points for these were trimmed off for better visualization. The segmentation accuracy for $\lambda=1$ was very low because the models were not trained for typical texture patterns at all. The distributions of the metrics can be seen in the box-whisker plots in Fig. 6, in which, only $\lambda=\{0,1, .6, .9\}$ are shown for concise visualization. The Wilcoxon signed-rank test was used to test the statistical significances between $\lambda=0$ and the other values. As shown in Fig. 5, the proposed method with $\lambda$ between 0 and 1 outperformed the results with $\lambda=0$. Larger $\lambda$ increases precision while decreasing recall. 
This is because larger $\lambda$ penalizes an unannotated region for being segmented as annotated label. The parameter of $\lambda=0.6$ balanced precision and recall well and achieved the best Dice score in our experiments.

The results of comparisons of the proposed method at $\lambda=0$ and $\lambda=0.6$ for each DLD class are shown in Fig. 7 and Table 2. As shown in Fig. 7, statistically significant improvement was observed for CON, GGO, HCM, and EMP. There was a statistically significant decrease in the Dice score for NOR, but the difference is smaller compared to other improvements, as shown in
Table 2. The Dice score for NOR was reduced, as not much room was left to improve the precision with NOR in the first place, while other DLDs improved their precision.

Figure 8 shows the confusion matrices of the segmentation results. The annotated pixels in the partially annotated dataset were used for the calculation of these matrices. Note that only annotated pixels were used for the calculation of the matrices, because the exact classes for unannotated pixels were unknown. Rows of the matrices were normalized; thus, diagonal elements of the

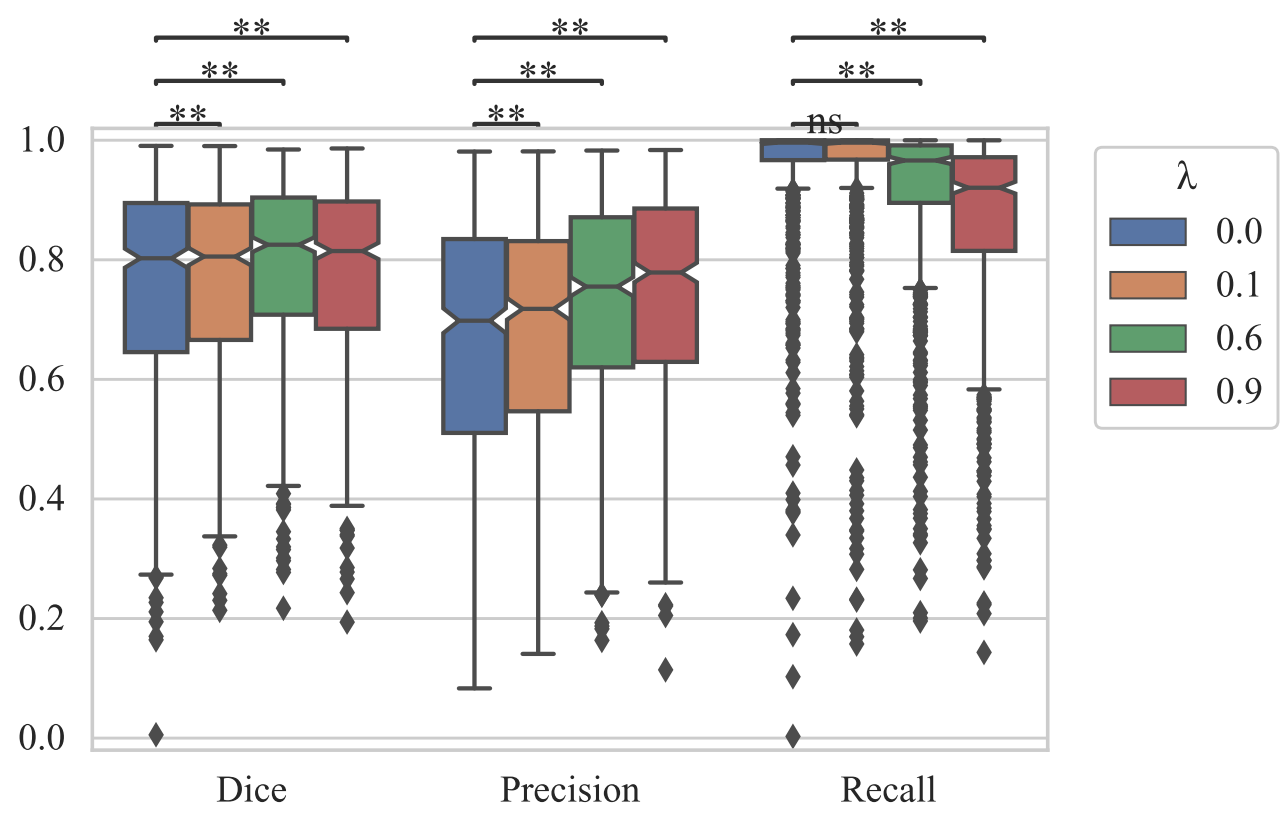

Fig. 6 Box-whisker plots for parameter comparison. Statistical significance is indicated by ${ }^{* *}(p<0.01)$.
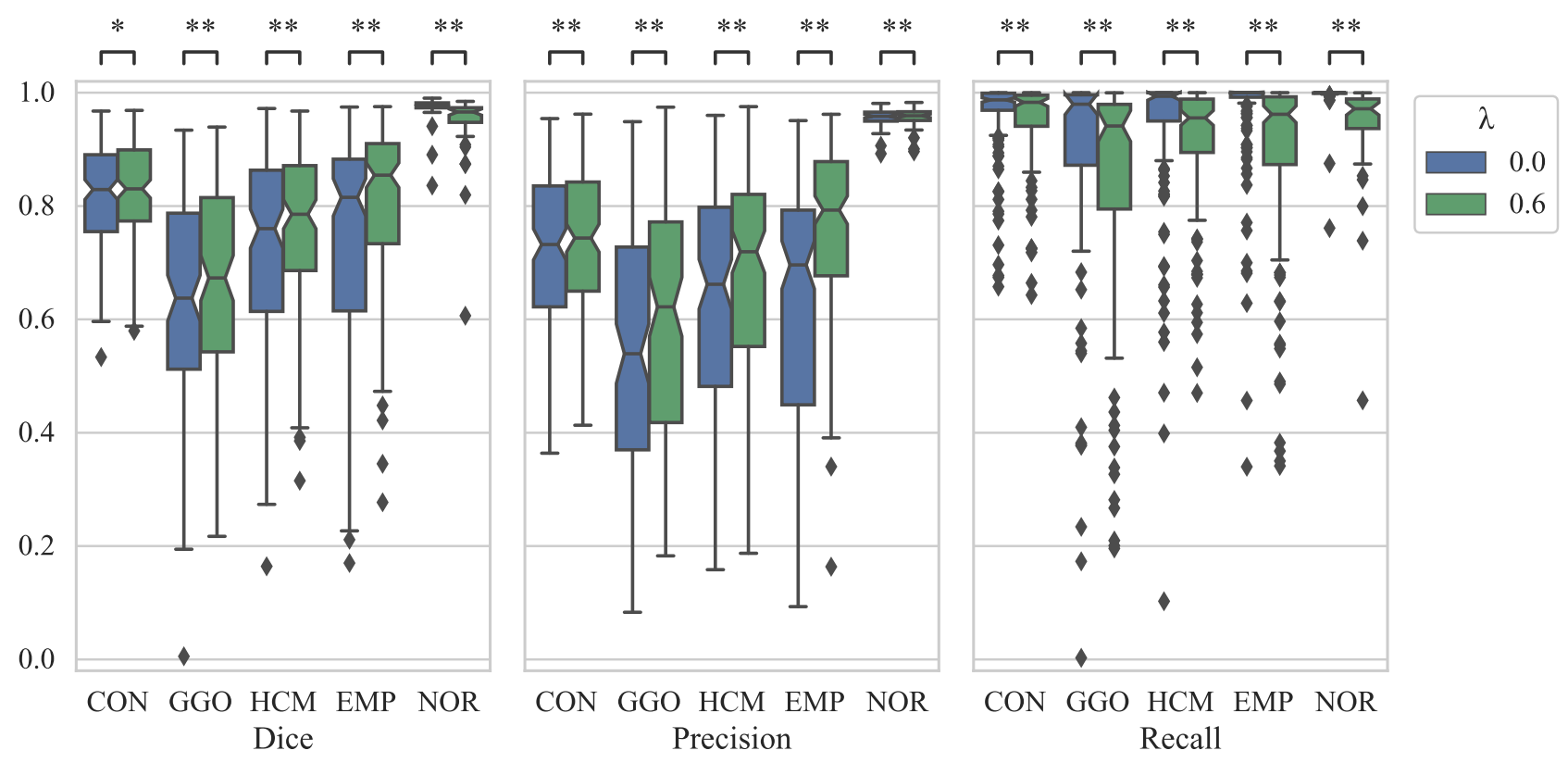

Fig. 7 Comparison between $\lambda=0$ and $\lambda=0.6$ for each DLD. Statistical significance is indicated by ${ }^{*}(p<0.05)$ or ${ }^{* *}(p<0.01)$. 
Table 2 Dice scores for each class.

\begin{tabular}{|c|c|c|c|c|c|c|}
\hline & $\mathrm{CON}$ & EMP & GGO & $\mathrm{HCM}$ & NOR & All \\
\hline \multicolumn{7}{|c|}{ mean (std) } \\
\hline$\lambda=0$ & $0.82(0.09)$ & $0.74(0.19)$ & $0.64(0.19)$ & $0.73(0.16)$ & $0.97(0.02)$ & $0.76(0.18)$ \\
\hline$\lambda=0.6$ & $0.82(0.09)$ & $0.81(0.14)$ & $0.67(0.17)$ & $0.75(0.15)$ & $0.95(0.06)$ & $0.79(0.15)$ \\
\hline \multicolumn{7}{|l|}{ median } \\
\hline$\lambda=0$ & 0.83 & 0.82 & 0.64 & 0.76 & 0.98 & 0.80 \\
\hline$\lambda=0.6$ & 0.83 & 0.85 & 0.67 & 0.79 & 0.97 & 0.83 \\
\hline
\end{tabular}

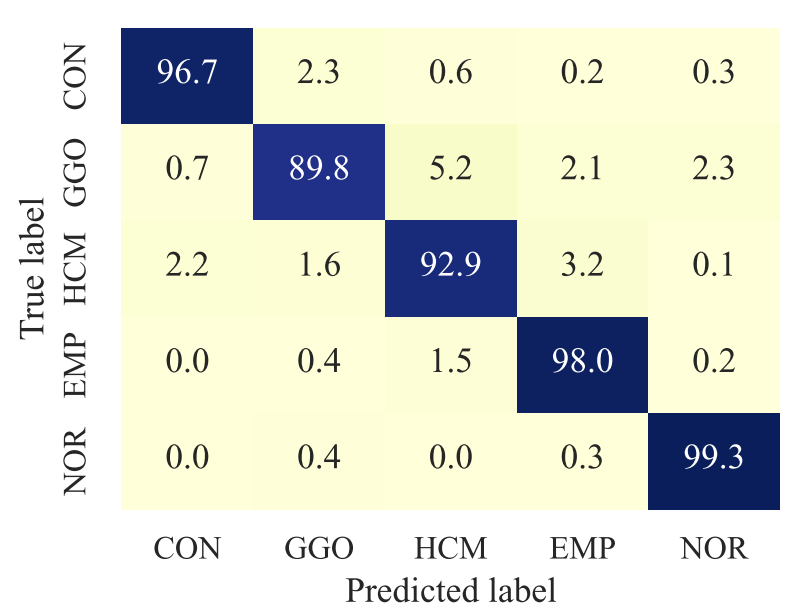

(a) $\lambda=0$

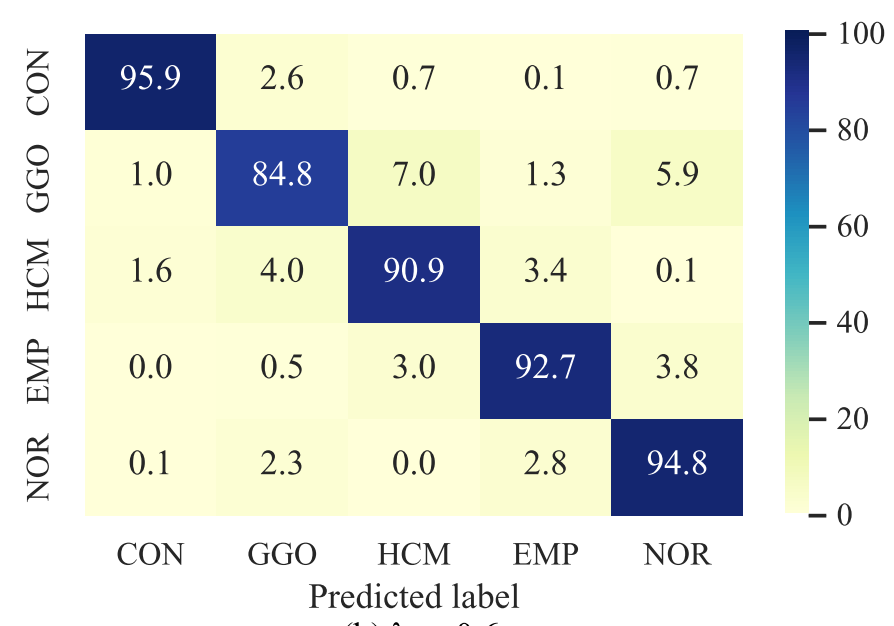

(b) $\lambda=0.6$

Fig. 8 Confusion matrices constructed using only annotated pixels in the partially annotated dataset. Row-wise normalizations are applied.

matrix represent recall. Figure 8(a) and (b) show similar patterns of misclassification. As diagonal elements of Fig. 8(a) and (b) show, recall of the results was lower when $\lambda=0.6$ compared to when $\lambda=0$, which is also shown in Fig. 7 and Table 2. Therefore, further analysis was conducted as shown below.

Figure 9 shows the typical segmentation results of each DLD class. The differences in the Dice scores between the results of $\lambda=0$ and $\lambda=0.6$ were used to choose the typical slices. The slice with the median difference in Dice score was chosen as a typical slice for each DLD class. As shown in Fig. 9, the area of representative DLD (especially GGO and EMP) was smaller at $\lambda=0.6$ than at $\lambda=0$, and fewer pixels of representative DLD were leaked to the non-representative DLD region, which reflected an improvement in precision.

In addition to the evaluation only using annotated pixels, we conducted additional evaluation using fully annotated images. For this evaluation, five slices were randomly chosen from each DLD class and all pixels in the lung fields of the chosen slices were manually annotated. Figure 10 shows the confusion matrices for the segmentation results at $\lambda=0$ and $\lambda=0.6$. Unlike confu- sion matrices shown in Fig. 8, these matrices were constructed using all pixels in the lung fields of fully annotated images. As shown in the bottom row of Fig. 10(a), a significant number of $\neg$ NOR pixels were falsely labeled as NOR at $\lambda=0$, whereas in Fig. 10(b), there were much less false NOR pixels at $\lambda=0.6$. As can be seen in the unannotated regions in Figs. 2 and 9, most of the unannotated pixels consisted of NOR pixels. Therefore, models trained at $\lambda=0.6$ was able to reduce the number of false NOR pixels. Table 3 shows the mean Dice scores. As shown in the table, $\lambda=0.6$ was also optimal in this evaluation. Figure 11 shows the segmentation results for fully annotated samples.

\section{DISCUSSION}

We developed a PSL approach and tested it using a partially annotated DLD dataset. The proposed method improved mean Dice score from 0.76 to 0.79 and from 0.73 to 0.82 in the evaluations using partially annotated dataset and fully annotated dataset, respectively. These improvements were credited to the improvements in precision as seen in Fig. 5, and were achieved with no extra cost for annotation. This means that training semantic 
(32)

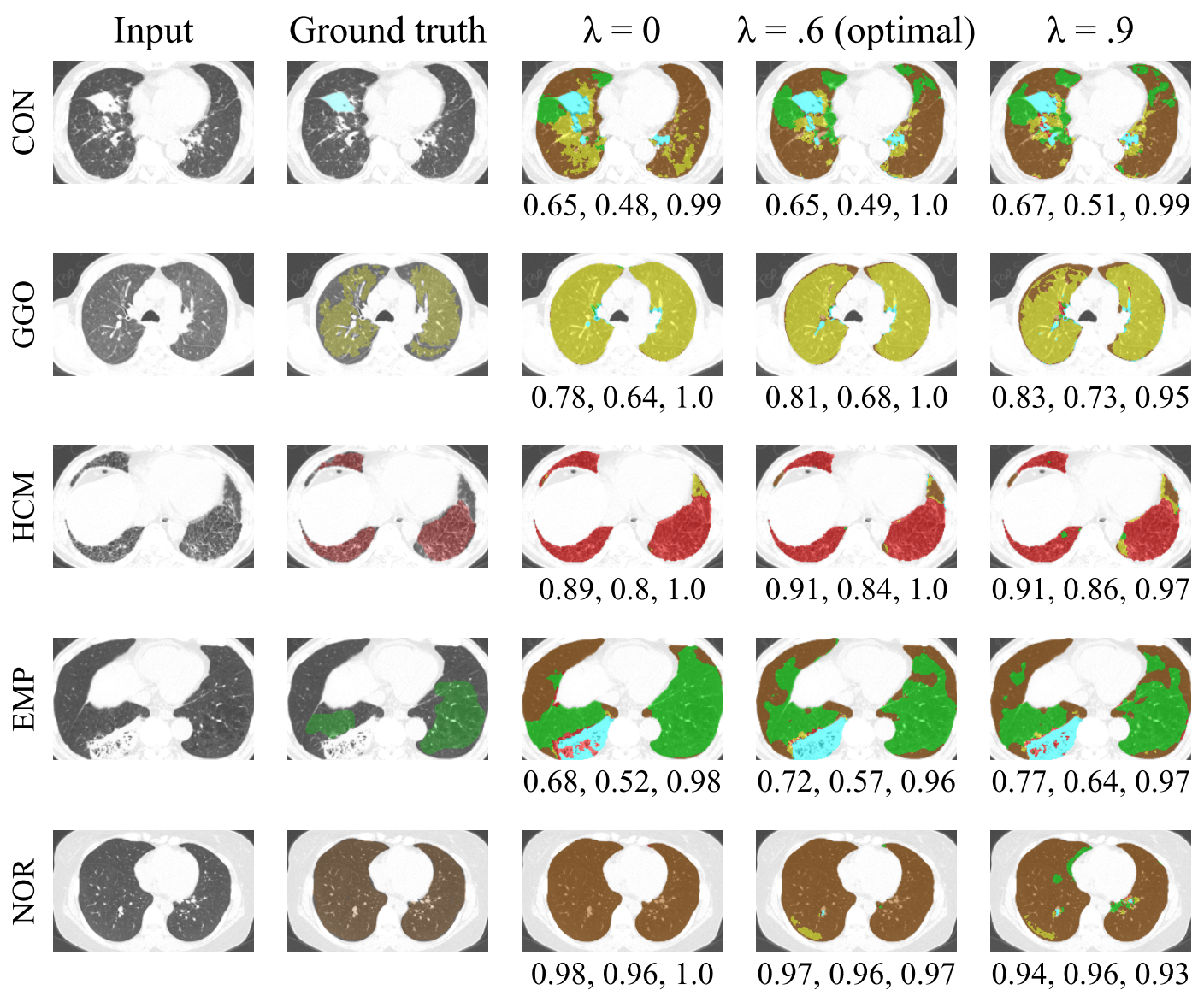

Fig. 9 Examples of segmentation results. The three numbers below an image indicate Dice score, precision, and recall.

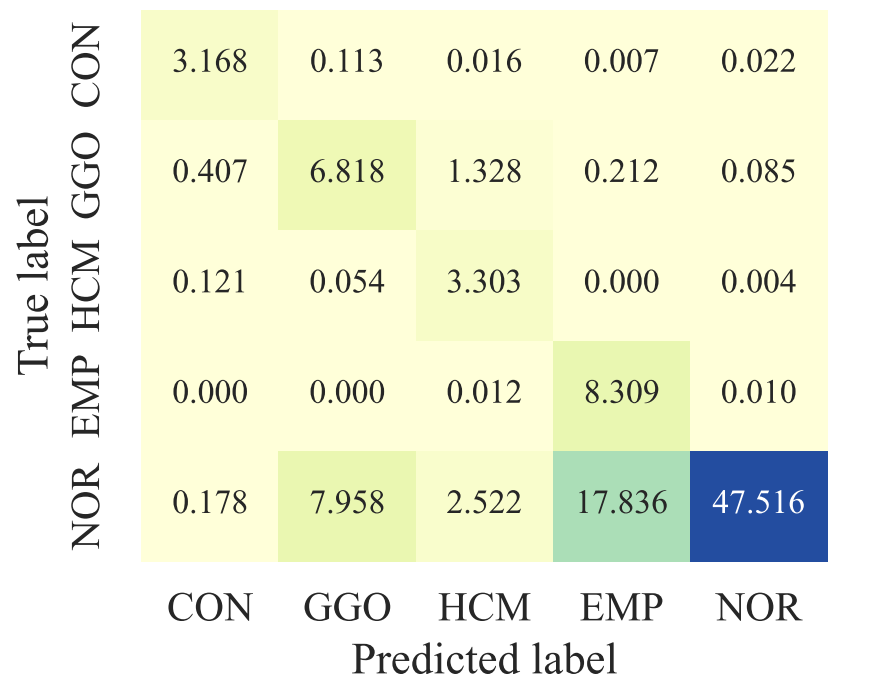

(a) $\lambda=0$

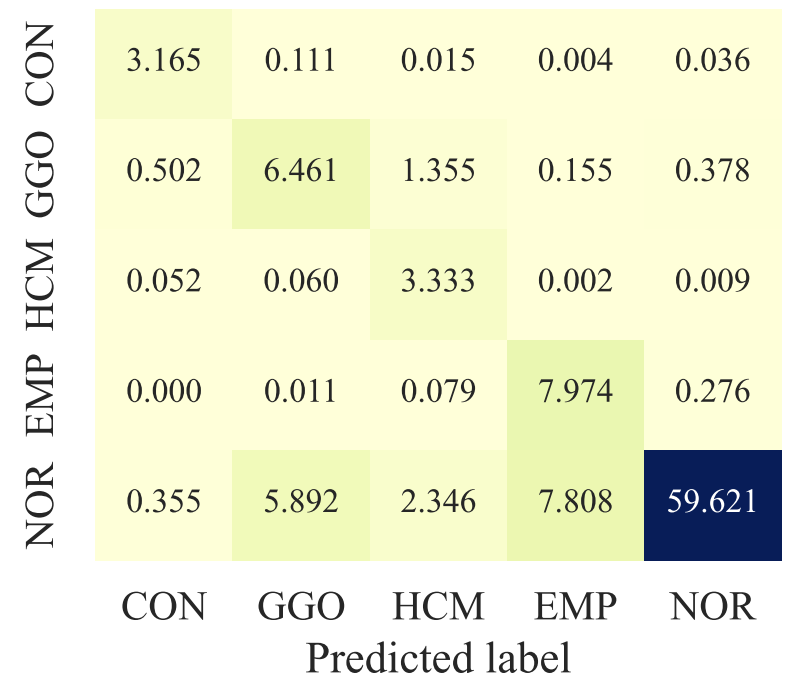

(b) $\lambda=.6$

Fig. 10 Confusion matrices calculated using all pixels in the lung fields of fully annotated images. Note that matrices are normalized so that the sum of the elements in a matrix is 100 .

Table 3 Mean Dice scores on fully annotated images.

\begin{tabular}{lrrrrrrrrrrr}
\hline$\lambda$ & 0 & 0.1 & 0.2 & 0.3 & 0.4 & 0.5 & 0.6 & 0.7 & 0.8 & 0.9 & 1 \\
\hline Dice & 0.726 & 0.801 & 0.791 & 0.819 & 0.813 & 0.813 & $\mathbf{0 . 8 2 3}$ & 0.816 & 0.801 & 0.816 & 0.004 \\
\hline
\end{tabular}



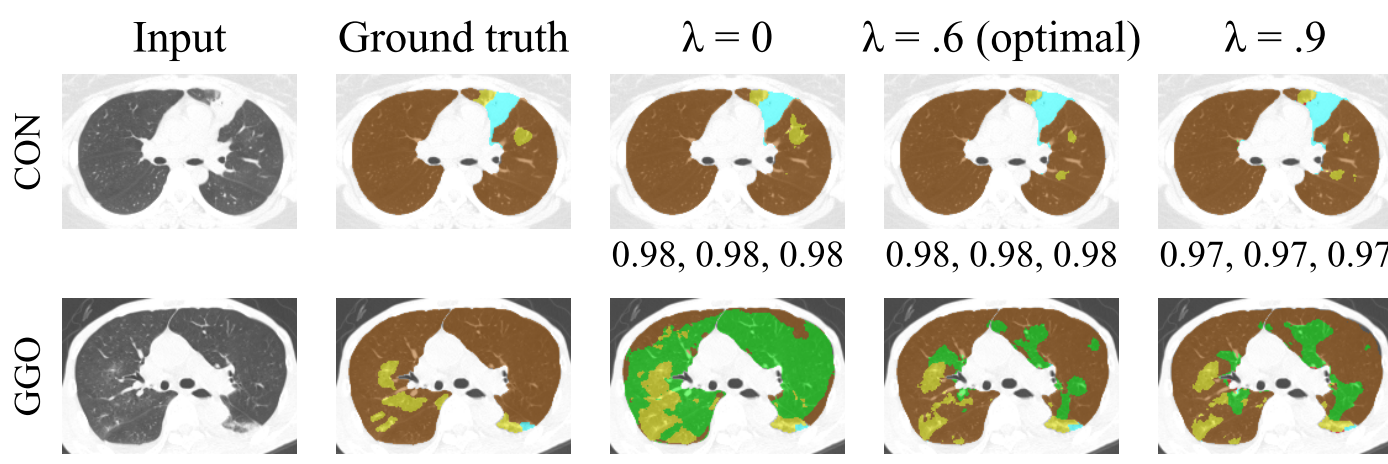

$0.98,0.98,0.98$
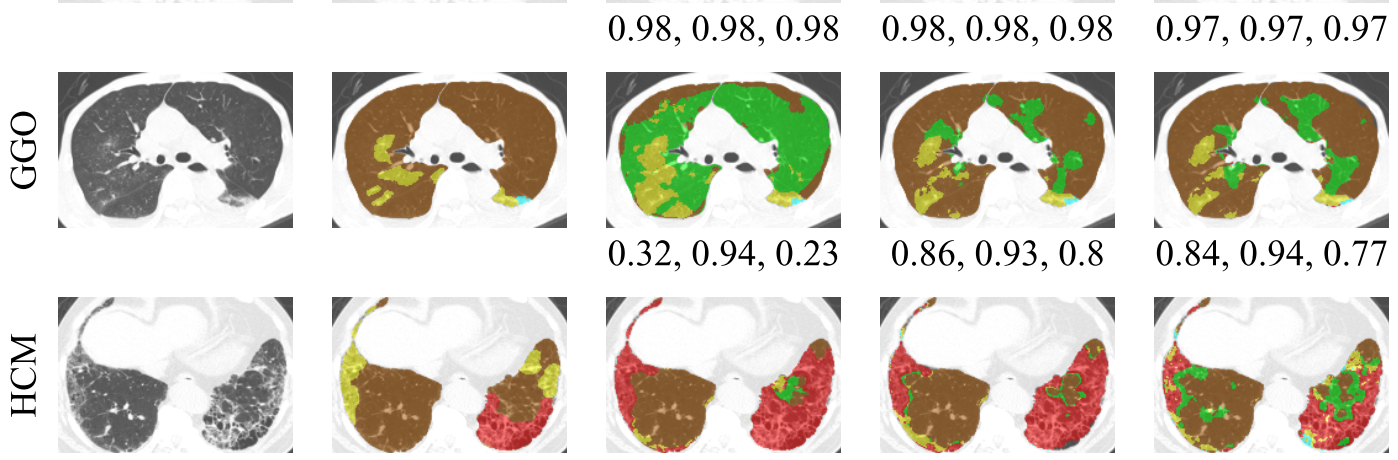

$0.86,0.93,0.8$

$0.84,0.94,0.77$
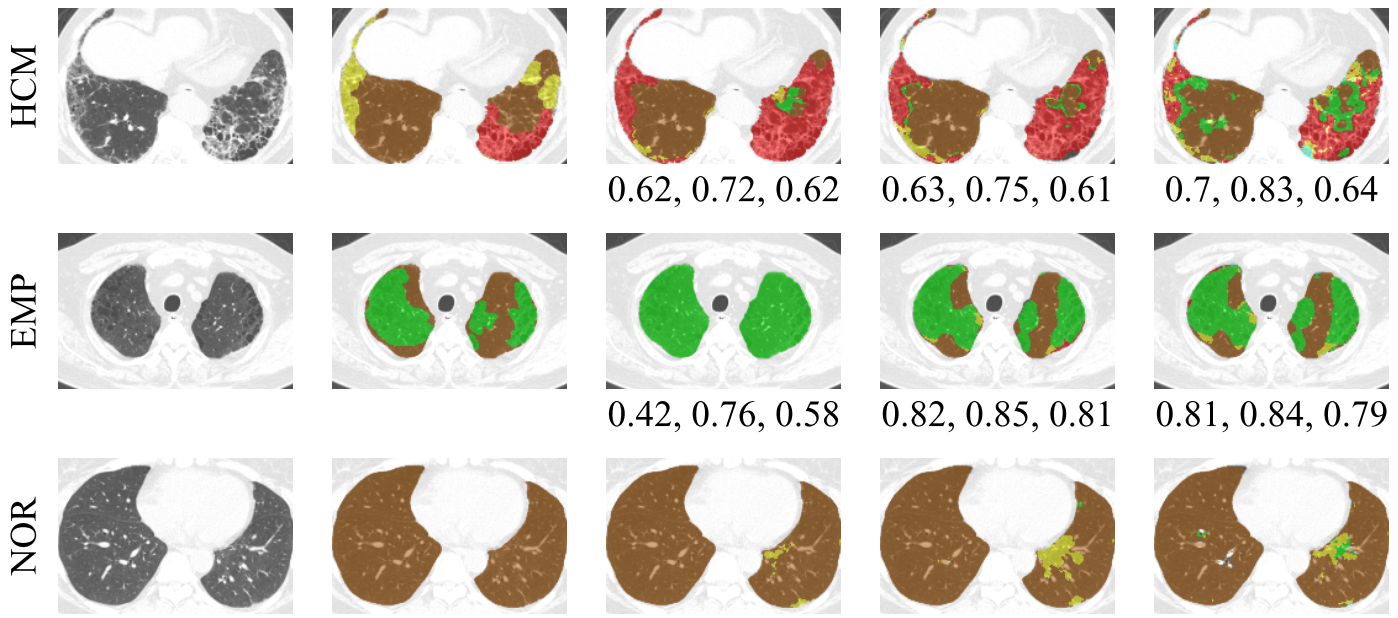

$0.99,1.0,0.98$
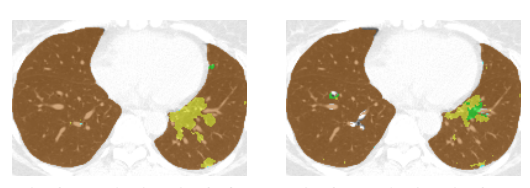

Fig. 11 Examples of segmentation results for fully annotated samples. The three numbers below an image indicate mean Dice score, precision, and recall.

segmentation models using the proposed method can improve accuracy compared with training methods that only used annotated pixels. Because PSL only requires partial annotation, active learning technique [34] can be used more easily to construct or update dataset. Low precision shown in Figs. 6 and 9 was likely due to the lack of full annotation in the training dataset and needs to be addressed in the future work.

In this study, we used partially annotated dataset in which only one class was annotated for an image. This setting was chosen to test the proposed method in the most challenging configuration. Moreover, publicly available datasets commonly have only single-class annotation, and the dataset used in this study is similar to a dataset created by combining such datasets. However, if the goal is to develop a multi-class segmentation model, preparing a dataset with at least two fully annotated images is more reasonable for achieving higher accuracy.

We conducted experiments with various values for the balancing parameter $\lambda$. As shown in Fig. 5, supervision using unannotated pixels helps improve the precision of the models. More specifically, the proposed method with optimal parameter significantly reduced the number of falsely labeled NOR pixels as shown in Fig. 10. This was because most of the unannotated (negative) pixels were actually NOR pixels, and training using these unannotated pixels helped the model distinguish NOR from non-NOR pixels. In our experiment, $\lambda=0.6$ was optimal regarding the Dice score, but the parameter can be tuned based on the preference for precision and recall. To the best of our knowledge, while we introduced the balancing parameter $\lambda$ to PSL for the first time in our previous study [30], Shi et al. [28] performed similar study that changed the balance of two terms in their loss function for multi-organ segmentation. Their experimental results showed similar curve as ours with peak accuracy at the optimal $\lambda$ of 0.67 , which was similar to our optimal parameter $(\lambda=0.6)$.

There are other possible approaches to realize multiclass segmentation using a partially annotated dataset. One approach is an ensemble model that combines multiple binary or subset classifier models using subsets of the dataset for the training of each sub-model. This approach, however, is expected to be less generalizable than the proposed PSL. Another possible approach is multi-task learning [35] that uses subsets of the dataset 
as different tasks, and trains one model with multiple output layers. Our future work will compare our method with other potential approaches $[11,12]$.

One of the limitations of this paper is that because the evaluation metrics were calculated mostly in annotated regions, the performance of the proposed PSL in unannotated regions was not fully studied, other than the precision shown in Fig. 7 and the evaluation using only a fraction of the dataset shown in Fig. 10 and Table 3. The lung tissue research consortium (LTRC) dataset [36] can be used for a more detailed study because the LTRC dataset provides fully annotated data.

A potential future direction is to combine PSL with other techniques such as transfer learning [20] to achieve even better accuracy. Another possibility is to combine other datasets in similar domain such as interstitial lung diseases from the University Hospital of Geneva [37] and the LTRC dataset [36], to test the possibility of improving accuracy using a combination of similar but slightly different datasets.

\section{Acknowledgement}

This work was supported by JSPS KAKENHI Grant Number 21H03840.

\section{Conflicts of interest}

The authors declare no conflicts of interest with any companies or commercial organizations per the definition of Japanese Society for Medical and Biological Engineering.

\section{Ethics declaration}

The study received Institutional Review Board approval at Yamaguchi University Hospital (H26-123-[1]-4). Need for informed consent was waived due to the retrospective nature of the study.

\section{References}

1. Widell J, Lidén M: Interobserver variability in high-resolution CT of the lungs. Eur J Radiol Open. 7, 100228, 2020.

2. Watadani T, Sakai F, Johkoh T, Noma S, Akira M, Fujimoto K, Bankier AA, Lee KS, Müller NL, Song J-W, Park J-S, Lynch DA, Hansell DM, Remy-Jardin M, Franquet T, Sugiyama Y: Interobserver variability in the CT assessment of honeycombing in the lungs. Radiology. 266(3), 936-944, 2013.

3. Gao M, Bagci U, Lu L, Wu A, Buty M, Shin H-C, Roth H, Papadakis GZ, Depeursinge A, Summers RM, Xu Z, Mollura DJ: Holistic classification of CT attenuation patterns for interstitial lung diseases via deep convolutional neural networks. Comput Methods Biomech Biomed Eng Imaging Vis. 6(1), 1-6, 2018.

4. Bermejo-Peláez D, Ash SY, Washko GR, San José Estépar R, Ledesma-Carbayo MJ: Classification of interstitial lung abnormality patterns with an ensemble of deep convolutional neural networks. Sci Rep. 10(1), 338, 2020.

5. Negahdar M, Coy A, Beymer D: An end-to-end deep learning pipeline for emphysema quantification using multi-label learning. Annu Int Conf IEEE Eng Med Biol Soc. 2019, 929-932, 2019.

6. Gao M, Xu Z, Lu L, Wu A, Nogues I, Summers RM, Mollura DJ: Segmentation label propagation using deep convolutional neural networks and dense conditional random field. 2016 IEEE 13th International Symposium on Biomedical Imaging (ISBI), 12651268, 2016.

7. Uemura T, Näppi JJ, Watari C, Hironaka T, Kamiya T, Yoshida H: Weakly unsupervised conditional generative adversarial network for image-based prognostic prediction for COVID-19 patients based on chest CT. Med Image Anal. 73, 102159, 2021.

8. Long J, Shelhamer E, Darrell T: Fully convolutional networks for semantic segmentation. Proc IEEE Comput Soc Conf Comput Vis Pattern Recognit. 3431-3440, 2015.

9. Ronneberger O, Fischer P, Brox T: U-net: Convolutional networks for biomedical image segmentation. Med Image Comput Assist Interv. 234-241, 2015.

10. Mabu S, Obayashi M, Kuremoto T, Hashimoto N, Hirano Y, Kido S: Unsupervised class labeling of diffuse lung diseases using frequent attribute patterns. Int J CARS. 12(3), 519-528, 2017.

11. Fidon L, Aertsen M, Emam D, Mufti N, Guffens F, Deprest T, Demaerel P, David AL, Melbourne A, Ourselin S, Deprest J, Vercauteren T: Label-set loss functions for partial supervision: application to fetal brain 3D MRI parcellation. Medical Image Computing and Computer Assisted Intervention MICCAI 2021, 647-657, 2021.

12. Peng J, Wang Y: Medical image segmentation with limited supervision: a review of deep network models. IEEE Access. 9, 36827-36851, 2021.

13. Anthimopoulos M, Christodoulidis S, Ebner L, Geiser T, Christe A, Mougiakakou S: Semantic segmentation of pathological lung tissue with dilated fully convolutional wetworks. IEEE J Biomed Health Inf. 23(2), 714-722, 2019.

14. Chapelle O, Scholkopf B, Zien A Eds.: Semi-supervised learning. IEEE Trans Neural Networks. 20(3), 542-542, 2009.

15. Tajbakhsh N, Jeyaseelan L, Li Q, Chiang JN, Wu Z, Ding X: Embracing imperfect datasets: a review of deep learning solutions for medical image segmentation. Med Image Anal. 63, 101693, 2020.

16. Wang C, Moriya T, Hayashi Y, Roth H, Lu L, Oda M, Ohkubo H, Mori K: Weakly-supervised deep learning of interstitial lung disease types on CT images. Medical Imaging 2019: ComputerAided Diagnosis. 10950, 373-379, 2019.

17. Laradji I, Rodriguez P, Manas O, Lensink K, Law M, Kurzman L, Parker W, Vazquez D, Nowrouzezahrai D: A weakly supervised consistency-based learning method for COVID-19 segmentation in CT images. Proc IEEE/CVF Winter Conf Appl Comput Vision. 2453-2462, 2021.

18. Shin H-C, Roth HR, Gao M, Lu L, Xu Z, Nogues I, Yao J, Mollura D, Summers RM: Deep convolutional neural networks for computer-aided detection: CNN architectures, dataset characteristics and transfer learning. IEEE Trans Med Imaging. 35(5), 1285-1298, 2016.

19. Chen S, Ma K, Zheng Y: Med3D: Transfer Learning for 3D Medical Image Analysis. 2019.

20. Mabu S, Atsumo A, Kido S, Kuremoto T, Hirano Y: Investigating the effects of transfer learning on ROI-based classification of chest CT images: a case study on diffuse lung diseases. J Sign Process Syst. 92(3), 307-313, 2020. 
21. Christodoulidis S, Anthimopoulos M, Ebner L, Christe A, Mougiakakou S: Multisource transfer learning with convolutional neural networks for lung pattern analysis. IEEE J Biomed Health Inf. 21(1), 76-84, 2017.

22. Fang X, Yan P: Multi-organ segmentation over partially labeled datasets with multi-scale feature abstraction. IEEE Trans Med Imaging. 39(11), 3619-3629, 2020.

23. Zhang J, Xie Y, Xia Y, Shen C: DoDNet: Learning to segment multi-organ and tumors from multiple partially labeled datasets. arXiv:2011.10217 [cs], 2020.

24. Dmitriev K, Kaufman AE: Learning multi-class segmentations from single-class datasets. 2019 IEEE/CVF Conf Comput Vision Pattern Recogn (CVPR). 9493-9503, 2019.

25. Roulet N, Slezak DF, Ferrante E: Joint learning of brain lesion and anatomy segmentation from heterogeneous datasets. Proc 2nd Int Conf Med Imaging Deep Learning. 401-413, 2019.

26. Zhou Y, Li Z, Bai S, Wang C, Chen X, Han M, Fishman E, Yuille AL: Prior-aware neural network for partially-supervised multiorgan segmentation. Proc IEEE/CVF Int Conf Comput Vision. 10672-10681, 2019.

27. Dorent R, Booth T, Li W, Sudre CH, Kafiabadi S, Cardoso J, Ourselin $\mathrm{S}$, Vercauteren T: Learning joint segmentation of tissues and brain lesions from task-specific hetero-modal domain-shifted datasets. Med Image Anal. 67, 101862, 2021.

28. Shi G, Xiao L, Chen Y, Zhou SK: Marginal loss and exclusion loss for partially supervised multi-organ segmentation. Med Image Anal. 70, 101979, 2021.

29. Liu X, Yuan Q, Gao Y, He K, Wang S, Tang X, Tang J, Shen D: Weakly supervised segmentation of COVID19 infection with scribble annotation on CT images. Pattern Recogn. 122, 108341, 2022.

30. Suzuki Y, Yamagata K, Yanagawa M, Kido S, Tomiyama N: Weak supervision in convolutional neural network for semantic segmentation of diffuse lung diseases using partially annotated dataset. Medical Imaging 2020: Computer-Aided Diagnosis, 11314, 113142A, 2020.

31. Çiçek Ö, Abdulkadir A, Lienkamp SS, Brox T, Ronneberger O: 3D U-Net: Learning dense volumetric segmentation from sparse annotation. Med Image Comput Assist Interv. 424-432, 2016.

32. Shorten C, Khoshgoftaar TM: A survey on image data augmentation for deep learning. J Big Data. 6(1), 60, 2019.

33. Kingma DP, Ba J: Adam: A method for stochastic optimization. arXiv preprint arXiv:1412.6980, 2014.

34. Yang L, Zhang Y, Chen J, Zhang S, Chen DZ: Suggestive annotation: a deep active learning framework for biomedical image segmentation. Med Image Comput Assisted Interv. 399-407, 2017.

35. Amyar A, Modzelewski R, Li H, Ruan S: Multi-task deep learning based CT imaging analysis for COVID-19 pneumonia: Classification and segmentation. Comput Biol Med. 126, 104037, 2020.

36. Bartholmai B, Karwoski R, Zavaletta V, Robb R, Holmes DRI: The Lung Tissue Research Consortium: An extensive open database containing histological, clinical, and radiological data to study chronic lung disease. Insight J. 2006.

37. Depeursinge A, Vargas A, Platon A, Geissbuhler A, Poletti P-A, Müller H: Building a reference multimedia database for interstitial lung diseases. Comput Med Imaging Graph. 36(3), 227-238, 2012.

\section{Yuki Suzukı}

Yuki SuzUKI received his B.S. degree in Engineering and M.S. degrees in Information Science from Osaka University in 2012 and 2014 respectively. From 2014 to 2019, he worked as a research assistant in Nara Institute of Science and Technology. He is currently a Specially Appointed Assistant

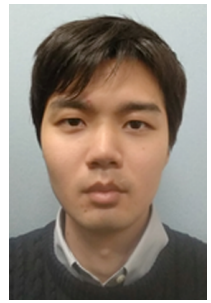
Professor in the Department of Artificial Intelligence Diagnostic Radiology, Osaka University Graduate School of Medicine. His research interests include medical image analysis, computational anatomy, and image processing.

\section{Shoji KIDo}

Shoji KIDo received his M.D. degree from Osaka University in 1988. He received his Ph.D. degrees in Medicine and Information Science from Osaka University in 1992 and 1999, respectively. He was a professor in the Department of Computer Science and Systems Engineering at Yamaguchi Uni-

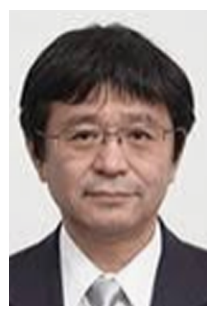
versity from 1999 to 2006, in the Department of Applied Medical Engineering Science, Graduate School of Medicine, Yamaguchi University from 2006 to 2016, and in the Division of Information Sciences and Engineering, Graduate School of Sciences and Technology for Innovation, Yamaguchi University from 2016 to 2019. He is currently a professor in the Department of Artificial Intelligence Diagnostic Radiology, Osaka University Graduate School of Medicine. His research interests include chest radiology, and computer-aided diagnosis based on artificial intelligence. He is a member of Japan Radiological Society, Radiological Society of North America, Japanese Society for Medical and Biological Engineering, Japanese Society of Medical Imaging Technology, Institute of Electronics, Information and Communication Engineers Medical imaging.

\section{Shingo MABU}

Shingo MABU received his B.E. and M.E. degrees from Kyushu University, Japan, in 2001 and 2003, respectively, and his Ph.D. degree from Waseda University, Japan, in 2006. From 2006 to 2007, he was a visiting lecturer at Waseda University, and from 2007 to 2012, he was an Assistant Professor

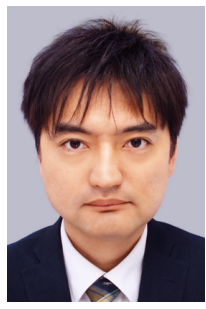
at the same university. From 2012 to 2017, he was an Assistant Professor at Yamaguchi University, Japan, and from 2017 to 2020, he was an Associate Professor at the same university. Since 2020, he has been a Professor at the same university. His research interests include machine learning and its applications. 


\section{Masahiro Yanagawa}

Masahiro YanAGawa received his degrees of M.D. and $\mathrm{Ph} . \mathrm{D}$. in Medicine from Osaka University in 2001 and 2009, respectively. In 2001, he started his carrier as a medical doctor in Osaka University Hospital. In 2012, he was an assistant professor in the department of Radiology, Osaka University

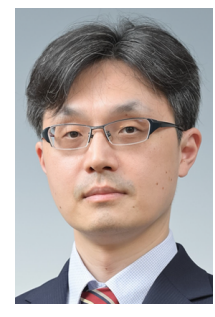

Graduate School of Medicine. He worked as a visiting research fellow in the department of Radiology, Stanford University, U.S.A in 2013. From 2019, he is an associate professor in the department of Radiology, Osaka University Graduate School of Medicine. His research interests include chest radiology (focused on thoracic oncology such as lung cancer, esophageal cancer, and mediastinal tumors), and computeraided diagnosis with and without artificial intelligence. He is a member of Japan Radiological Society, Radiological Society of North America, Japanese, Japan Lung Cancer Society, Japanese Society of Nuclear Medicine, Japanese Association for Research on the Thymus, Japanese Society of CT Screening, Japanese Respiratory Society, and Japanese Society for Magnetic Resonance in Medicine. In addition, he is a scientific editorial board member of chest section, European Radiology.

\section{Noriyuki TomiYama}

Noriyuki TомiYama received his degrees of M.D. and Ph.D. in Medicine from Osaka University in 1987 and 1993 respectively. In 1987, he started his carrier as a medical doctor in Osaka University Hospital. After he graduated from Osaka University Graduate School of Medicine, he held the vari-

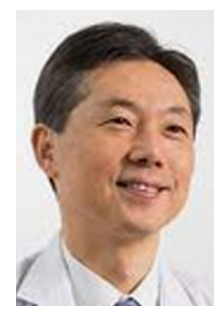
ous posts such as Assistant professor and Associate professor in the Department of Radiology of the school. He worked as a research fellow in Vancouver General Hospital, Canada in 1999, and as a staff in Ehime National Hospital from 2000 to 2002. From 2010, he is the Professor and Chairman of the Department of Radiology, Osaka University Graduate School of Medicine. Dr. Tomiyama is a Vice President of Japan Radiological Society and the Presidential Elect of Asian Oceanian Society of Radiology. In addition, he is a board member of Japanese Society of Thoracic Radiology and Japan Radiology Congress and a member of Radiological Society of North America, Fleischner Society, International Society for Strategic Studies in Radiology, and Japanese Medical Specialty Board.

\section{Yoshinobu Sato}

Yoshinobu SATo received his B.S., M.S. and Ph.D degrees in Information and Computer Sciences from Osaka University, Japan in 1982, 1984, 1988, respectively. From 1988 to 1992, he worked at the NTT Human Interface Laboratories. In 1992, he joined Osaka University Medical School. From

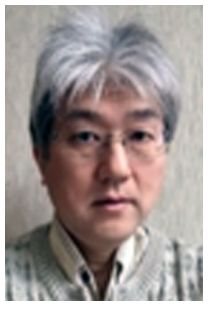
1996 to 1997, he was a Visiting Research Fellow at the Surgical Planning Laboratory, Harvard Medical School and Brigham and Women's Hospital. In 1999, he became an Associate Professor at Osaka University Graduate School of Medicine. From 2014, he is a Professor at Nara Institute of Science and Technology (NAIST). His research interests include medical image analysis, computer assisted surgery, and computational anatomy. Dr. Sato is an Editorial Board member of Medical Image Analysis journal and International Journal of Computer Assisted Radiology and Surgery (IJCARS) journal. He was Program Chair of the 16th International Conference on Medical Image Computing and Computer Assisted Intervention (MICCAI 2013). 